\title{
Studies of prokaryotic Type II topoisomerase drug inhibition
}

Mark R. Sanderson $^{1}$, Ivan Laponogov ${ }^{1}$, Xiaosu Pan ${ }^{2}$, Dennis A. Veselkov ${ }^{1}$, Isabelle M-T. Crevel ${ }^{2}$, Jogitha Selvarajah ${ }^{2}$, Art Branstrom $^{3}$, Ryan Cirz ${ }^{4}$, Heinz E. Moser ${ }^{5}$, L. Mark Fisher ${ }^{2}$

${ }^{1}$ Randall Division of Cell and Molecular Biophysics, 3rd Floor New Hunt's House, Division of Medical and Life Sciences, King's College, Guys Campus, London Bridge, London, U.K., ${ }^{2}$ Molecular and Clinical Science Institute, St. George's, University of London, Cranmer Terrace, London, U.K., ${ }^{3}$ PTC Pharmaceuticals, South Plainfield, New Jersey, United States, ${ }^{4}$ Achaogen Pharmaceuticals Inc., San Francisco, California, USA, ${ }^{5}$ Novartis Institute for Biomedical Research, Emerville, California, USA E-mail: mark.sanderson@kcl.ac.uk

Type II topoisomerases perform essential roles in DNA replication, chromosome segregation, and recombination and are important antibacterial and anticancer targets [1]. Bacterial drug resistance is a growing and now widely recognised threat and the limited number of new antibacterials developed in the recent years is a matter of serious concern. One of the approaches to combat this growing threat is to investigate deeply the mechanisms of action of currently available antibacterials, as well as studying the way that bacteria are developing drug resistance and may potentially in the future develop drug resistance to known drugs. This knowledge will in turn be used in the rational drug design and the general development of the appropriate molecular frameworks for combating bacterial infections, while at the same time keeping the negative side effects of the drugs to an acceptable minimum. This is especially important when both bacteria and humans share similar drug targets, such as is the case for type II topoisomerases (in humans topo IIa and topo II $\beta$ are targeted by anti-cancer drugs i.e. doxorubicin and etoposide), whereas in prokaryotes Gyrase and topoisomerase IV (topoIV) may be targeted by quinolones and quinazolinediones.

Type II topoisomerases which are involved in regulation of the DNA supercoiling in both bacteria (gyrase) and eukaryotes and also in decatenation of bacterial daughter chromosomes during cell division (topo IV). Type II topoisomerases perform their biological action by binding double stranded DNA (called G-segment or Gate-DNA), temporarily cleaving it and passing another double stranded DNA (called T-segment) in the ATP-assisted process via the cleavage region thus changing the linking number in steps of \pm 2 . After that the G-segment is resealed and released. Quinolones, doxorubicin and etoposide are found to be able to disrupt this process ultimately resulting in the cell death (hence their anti-bacterial or anti-cancer action). Here we present our studies on the topo II catalytic cycle and the protein-DNA-drug interactions which are involved in the action of clinically used and newly developed quinolone antibacterials [2-3].

[1] Hauk, G. \& Berger, J.M. (2016). Curr. Opin. Struct. Biol. 36, 85-96.

[2] Veselkov, D.A. et al. (2016). Acta Cryst. D Structural Biology 72, 488-496.

[3] Laponogov, I. et al. Open Biology 6(9), 160157.

Keywords: drug development, topoisomerase II, bacterial enzyme targeting 